SFB

Benchmarking evolutionary multiobjective optimization algorithms

823

Olaf Mersmann, Heike Trautmann, Boris Naujoks, Claus Weihs

Nr. 3/2010

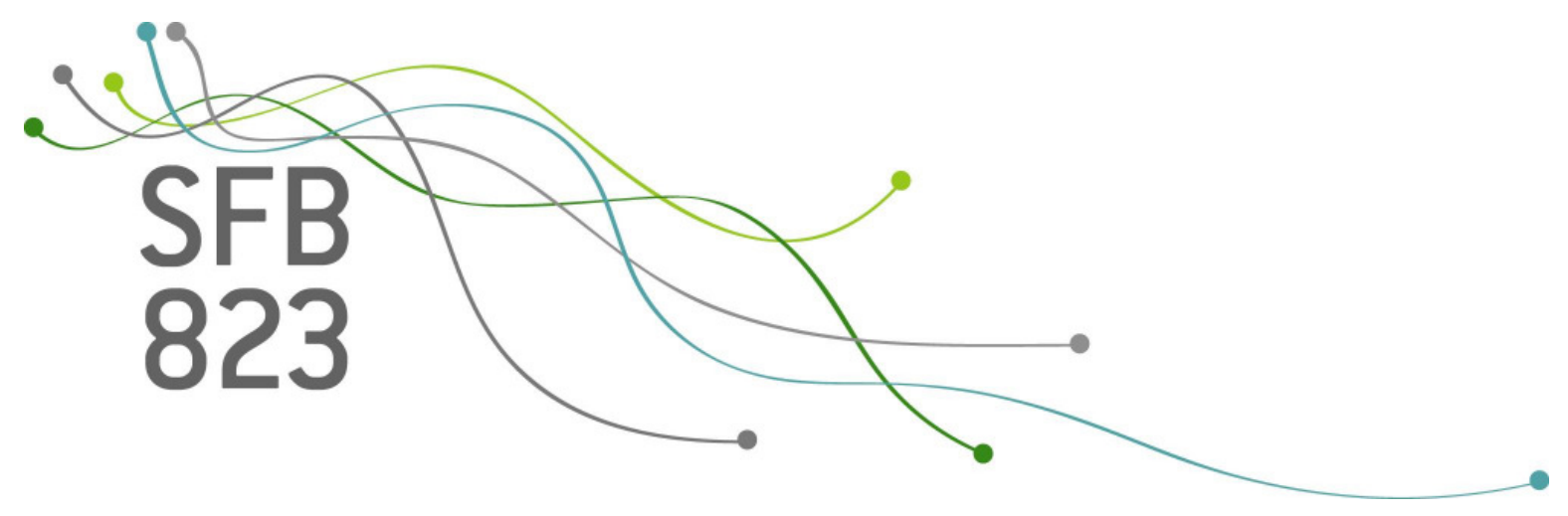





\title{
Benchmarking Evolutionary Multiobjective Optimization Algorithms
}

\author{
Olaf Mersmann ${ }^{1}$, Heike Trautmann ${ }^{1}$, Boris Naujoks $^{2}$ and Claus Weihs ${ }^{1}$ \\ ${ }^{1}$ Statistics Faculty, TU Dortmund \\ ${ }^{2}$ Log! in GmbH, Schwelm \\ e-mail: \{mersmann;trautmann;weihs\}@ statistik.tu-dortmund.de, \\ boris.naujoks@login-online.de
}

February 2010

\begin{abstract}
:
Choosing and tuning an optimization procedure for a given class of nonlinear optimization problems is not an easy task. One way to proceed is to consider this as a tournament, where each procedure will compete in different 'disciplines'. Here, disciplines could either be different functions, which we want to optimize, or specific performance measures of the optimization procedure. We would then be interested in the algorithm that performs best in a majority of cases or whose average performance is maximal.

We will focus on evolutionary multiobjective optimization algorithms (EMOA), and will present a novel approach to the design and analysis of evolutionary multiobjective benchmark experiments based on similar work from the context of machine learning. We focus on deriving a consensus among several benchmarks over different test problems and illustrate the methodology by reanalyzing the results of the CEC 2007 EMOA competition.
\end{abstract}

\section{Introduction}

Comparing the outcome of competitors in multiple disciplines is a complex task. It always depends on the considered ranking and consensus methods applied. The task to judge the outcome of evolutionary algorithms on different test problems considering multiple runs falls into this area. It becomes even harder if one wants to compare evolutionary multiobjective optimization algorithms as different performance measures have to be taken into account as well.

The development of such algorithms has evolved into a large research field during the last 20 years. Recently there has been work on the systematic evaluation and benchmarking of the 
rapidly increasing number of EMOA [1]. This focuses on the systematic comparison of pairs and groups of algorithms using statistical tests and graphical representations of the algorithms' performance. Furthermore, there have been several competitions for EMOA where the resulting rankings were analyzed using an ad hoc approach.

Motivated by the work of Hornik and Meyer [2] on the systematic benchmarking of classification algorithms, we will derive a suitable benchmarking framework to statistically compare EMOA. All necessary functions are implemented in an R package named emoa [3]. The ranking and consensus methods stem from the R package relations [4].

In Section 2 we will review relations, rankings and orders. Then we will introduce benchmark scenarios in Section 3, define ranking operators for these scenarios and then derive a consensus ranking from these individual benchmark scenario rankings. In Section 6 we apply the methods previously proposed to a dataset from the CEC 2007 EMOA competition. Since there cannot be a universal best consensus [5], we find different outcomes for the competition depending on the quality indicator, the ranking method and the consensus method applied. We close by giving a short summary and suggestions for future research in section 7 .

\section{Relations, orders and rankings}

We successively refine the general term relation ([6]) until we arrive at a formal definition for a ranking.

Definition 1 A binary relation $R$ is given by the pair $\langle D(R), G(R)\rangle$ where $D(R)=\left(X_{1}, X_{2}\right)$ is the domain of the relation $R$ and $G(R)$ the graph of $R$. A tuple $x \in D(R)$ is said to be contained in the relation $R$ if and only if (iff) it is an element of the graph $G(R)$. Let $s_{i}$ be the cardinality of $X_{i}$. Then $s=\left(s_{1}, s_{2}\right)$ is called the size of $R$. A binary relation $R$ is called an endorelation iff $X_{1}=X_{2}$. If all the $s_{i}$ are finite, the graph $G(R)$ can be represented by a matrix $\vec{I}(R)$ with size $s$. It is called the incidence matrix of the relation $R$ and for any $x \in D(R)$ $\vec{I}_{x}=1$ iff $x$ is contained in $G(R)$.

We will assume that all elements of our domain $D$ are finite sets. A relation $R$ can therefore always be uniquely represented by its incidence array $\vec{I}(R)$.

Definition 2 Let $R$ be an endorelation with domain $D(R)=(X, X)$. Then it is called

- reflexive if for all $x \in X, x R x$.

- transitive if for $x, y, z \in X, x R y \wedge y R z \Rightarrow x R z$. 
- antisymmetric if for $x, y \in X, x R y \wedge y R x \Rightarrow x=y$.

- complete if for all distinct $x, y \in X, x R y \vee y R x$.

Definition 3 A weak order is a complete, reflexive and transitive endorelation. A partial order is a reflexive, antisymmetric and transitive endorelation. A linear (or total) order is a antisymmetric weak order.

All orders have the important property of being transitive.

Definition 4 A ranking $R$ of a set of items $\mathcal{A}=\left\{a_{1}, a_{2}, \ldots, a_{k}\right\}$ is a weak order over the set $\mathcal{A}$. For $x, y \in \mathcal{A}$ we say $x$ is better than $y$ and denote this by $x \succ y$ iff $y R x$ and not $x R y$. If $x R y$ and $y R x$, then we say $x$ and $y$ are tied and denote this by $x \sim y$.

A strict ranking $R$ of a set $\mathcal{A}=\left\{a_{1}, a_{2}, \ldots, a_{k}\right\}$ is a linear order over the set $\mathcal{A}$. For $x, y \in \mathcal{A}$ we say $x$ is preferred over $y(x \succ y)$ iff $y R x$.

In the following we will denote the ranking, as well as the relation by $R$ and use $\succ$ for the corresponding binary operator between elements from the domain of $R$. If applicable, we will use the shorthand form of writing $x \succ y \succ z$.

\section{Benchmark Scenarios}

Informally, we are interested in the best algorithm from a set of EMOA. At first we need to limit ourselves to a domain of optimization problems. A crucial and very important task is the definition of better.

Definition 5 Given a set of possible parameters $\mathcal{X}$ and $d$ functions $f_{1}, \ldots, f_{d}$ with $f_{i}: \mathcal{X} \rightarrow \mathbb{R}$ for $i \in\{1, \ldots, d\}$, a multiobjective optimization problem is given by

$$
\arg \min _{\vec{x} \in \mathcal{X}} \tilde{f}_{i}(\vec{x}) \quad i=1, \ldots, d
$$

where $\tilde{f}_{i}=f_{i}$ if $f_{i}$ should be minimized and $\tilde{f}_{i}=-f_{i}$ if $f_{i}$ should be maximized.

For a performance analysis of EMOA [7] we need to pick a fixed quality indicator $I$ (e.g. Dominated Hypervolume, see [1] for an overview) which has to be maximized by assumption. If $I$ is a binary quality indicator we assume that we use a given common reference set. So we can always take $I$ to be a unary quality indicator for which greater values mean higher worth. 
So given a quality indicator $I$, a multiobjective optimization problem $f$ and two EMOA $a_{1}$ and $a_{2}$ we wish to define a comparison operator $\succ$ which tells us if $a_{1}$ is as good as or better than $a_{2}$ where, by definition, $a \succ a$ for any algorithm $a$.

Definition 6 Given two algorithms $a_{1}$ and $a_{2}$, a fixed optimization problem $f$, quality indicator $I$ and a pairwise comparison operator $\succ$, we say $a_{1}$ is best iff $a_{1} \succ a_{2}$ and not $a_{2} \succ a_{1}$. Conversely, we say $a_{2}$ is best iff $a_{2} \succ a_{1}$ and not $a_{1} \succ a_{2}$. If both $a_{1} \succ a_{2}$ and $a_{2} \succ a_{1}$, we say $a_{1}$ and $a_{2}$ are tied.

This definition is similar to the definition of Pareto dominance.

Definition 7 Given two parameter settings $\vec{x}$ and $\vec{x}^{\prime}, \vec{x}$ dominates $\vec{x}^{\prime}\left(\vec{x} \succ \vec{x}^{\prime}\right)$ iff $\forall i \in\{1, \ldots, d\}$ :

$$
f_{i}(\vec{x}) \leq f_{i}\left(\vec{x}^{\prime}\right) \wedge \exists i \in\{1, \ldots, d\}: f_{i}(\vec{x})<f_{i}\left(\vec{x}^{\prime}\right)
$$

The problem with this definition is that it cannot be generalized to three or more algorithms. To see this, let us look at the set of possible results from all pairwise comparisons between three algorithms $a_{1}, a_{2}$ and $a_{3}$.

$$
\mathcal{A}=\left\{a_{1} \succ a_{2}, a_{1} \succ a_{3}, a_{2} \succ a_{1}, a_{2} \succ a_{3}, a_{3} \succ a_{1}, a_{3} \succ a_{2}\right\} .
$$

The implied $a_{i} \succ a_{i}$ entries have been ommited and we will assume that we have no tied entries for the moment.

The subset $\mathcal{A}^{\prime}=\left\{a_{1} \succ a_{2}, a_{1} \succ a_{3}, a_{2} \succ a_{3}\right\}$ could be shortened to $a_{1} \succ a_{2} \succ a_{3}$. So we have deduced a ranking of the three algorithms. In fact, we can say with some confidence, that $a_{1}$ is the best algorithm since it is better than any algorithm in a pairwise comparison.

However, we could just as well have gotten the result $\mathcal{A}^{\prime \prime}=\left\{a_{1} \succ a_{2}, a_{2} \succ a_{3}, a_{3} \succ a_{1}\right\}$, which does not induce an order on the algorithms since it contains a cycle. The only possible conclusion is that $a_{1}$ is equal to $a_{2}$ is equal to $a_{3}$. But this would mean that we should have also observed $a_{2} \succ a_{1}, a_{3} \succ a_{2}$ and $a_{1} \succ a_{3}$. In this case we have no real interpretation for the result set.

Generally, any subset of $\mathcal{A}$ defines a graph over $\left\{a_{1}, a_{2}, a_{3}\right\}$. We can therefore interpret our pairwise comparison operator as an endorelation over $\left\{a_{1}, a_{2}, a_{3}\right\}$. Knowing this, we can now use the definitions from Section 2 to derive desirable properties for our comparison operator, so that the corresponding endorelation is some type of order.

We know that the comparison operator is by definition reflexive and needs to be transitive. In the following sections we will develop several different comparison operators and show which properties they meet. In all cases, we will assume that there is some true underlying order of the algorithms. 


\section{Ranking}

We assume that we are given $k$ algorithms $a_{1}, \ldots, a_{k}$, a quality indicator $I$ and a multiobjective optimization problem $f$. If, given the optimization problem and the algorithm, our performance measure was fixed, we could easily obtain a ranking operator by simply defining

$$
a_{1} \succ a_{2} \Longleftrightarrow I\left(a_{1}\right) \geq I\left(a_{2}\right)
$$

However, since we know that the $a_{i}$ are randomized search algorithms, it follows that given $f$ and $a_{i}, I$ follows some unknown distribution $L_{I \mid f, a_{i}}$. We will assume that $L_{I \mid f, a_{i}}$ is continuous and that if $I_{i}$ follows the distribution $L_{I \mid f, a_{i}}$ then the expected value $E(I)$ exists.

\subsection{Summary statistic based ranking}

Since $I$ follows some distribution, the simplest approach to order the $a_{i}$ is to calculate a summary statistic of the distribution and then use the greater than or less than relation to derive a linear order. This approach is nice in that we know that the result will always be a linear order of the algorithms. However, it assumes that the summary statistic accurately captures the information from each distribution we wish to be included in the ranking.

Since we assume that the true distribution is unknown, we need to estimate our chosen summary statistic based on a random sample from the distribution. The result we obtain will always be a linear order, as we do not take into account any uncertainty in our estimates when constructing the ranking.

For the rest of this section, we will assume that we have a fixed budget of $n$ runs for each of the $k$ algorithms that we can afford. This means that we have a fixed sample of $n$ quality indicator values $I_{i, 1}, \ldots, I_{i, n}$ for each algorithm $a_{i}$. We will also assume that the runs are conducted in a manner that they result in an independent and identically distributed sample from the underlying distribution $L_{I \mid f, a_{i}}$.

\section{Mean ranking}

We define the mean ranking to be the linear order which ranks the $k$ algorithms $a_{1}, \ldots, a_{k}$ according to the average values of the quality indicator $\bar{I}_{i}$, using the greater than or equal relation $\geq$. We know that

$$
\bar{I}_{i}:=\frac{1}{n} \sum_{j=1}^{n} I_{i, j},
$$

is the unbiased estimator of the expected value of $I_{i}$ with the lowest variance. 


\section{Median ranking}

The median ranking is defined to be the linear order of the empirical medians of the quality indicator values. We can interpret the median as a compromise between average case quality and likely quality. We again use the $\geq$ relation to induce a linear order on the observed medians since, by definition, we want to maximize the value of the quality indicator.

\section{Maxi-Min ranking}

If we wanted to choose our algorithm to maximize the worst case quality, we would use the empirical minimum of our quality indicator values as the statistic to order. Similarly if we wanted to maximize the best case quality we would order the empirical maximum of the quality indicator values.

\section{Variance ranking}

The unbiased estimator with the lowest variance for the variance of $L_{I \mid f, a_{i}}$ is

$$
I_{i}^{v a r}:=\frac{1}{n-1} \sum_{j=1}^{n}\left(I_{i, j}-\bar{I}_{i}\right)^{2} .
$$

A low variance implies that we get consistent quality, thus now we want to minimize the summary statistic. So the variance ranking of our algorithms is given by the linear order of the $I_{i}^{s d}$ using the less than or equal relation $\leq$.

The proposed summary statistics are summarized in Table 1. The corresponding comparison operator can be constructed from the table by the following rule:

$$
a_{i} \succ a_{j} \Longleftrightarrow s_{i} R s_{j}
$$

The list of summary statistics and benchmarking scenarios is certainly not complete. Other comparison operators can be constructed based on the same principles.

\subsection{Relevance ranking}

All summary statistics based comparison operators do not take into account the variability of the underlying data. They do not consider how sure we can be that the difference between two summary statistics $s_{i}$ and $s_{j}$ is relevant and that their relation to each other is not reversed. In this section we will discuss two approaches to deal with this. The first one is based on expert 


\begin{tabular}{lll}
\hline Scenario & Summary statistic $\left(s_{i}\right)$ & $R$ \\
\hline Best case quality & $\max \left\{I_{i, 1}, \ldots, I_{i, n}\right\}$ & $\geq$ \\
Average case quality & $n^{-1} \sum_{j=1}^{n} I_{i, j}$ & $\geq$ \\
Median quality & $\operatorname{median}\left(I_{i, 1}, \ldots, I_{i, n}\right)$ & $\geq$ \\
Worst case quality & $\min \left\{I_{i, 1}, \ldots, I_{i, n}\right\}$ & $\geq$ \\
Consistent quality & $(n-1)^{-1} \sum_{j=1}^{n}\left(I_{i, j}-\bar{I}_{i}\right)^{2}$ & $\leq$ \\
\hline
\end{tabular}

Table 1: List of summary statistics and order relations $R$.

knowledge about the quality indicator, and the second one uses statistical hypothesis tests to judge if a difference is relevant.

If a domain expert can provide us with a relevant difference $\delta$ for our summary statistic, then we modify our decision rule from above to be

$$
\begin{aligned}
a_{i} \succ a_{j} & \Longleftrightarrow\left|s_{i}-s_{j}\right|>\delta \wedge s_{i} R s_{j}, \\
a_{i} \sim a_{j} & \Longleftrightarrow\left|s_{i}-s_{j}\right| \leq \delta .
\end{aligned}
$$

This rule is still transitive, reflexive and complete, however it lacks antisymmetry and therefore only induces a weak ordering on our set of algorithms.

The main drawback of this approach is that it assumes linearity of the scale. E.g. this is not the case for the hypervolume indicator $I_{H V}$ [8]. At the same time the difference does not take into account the variability, instead $\delta$ must be chosen large enough to account for any $s_{i}$ with a large variance. Alternatively, we are looking for the smallest significant difference which we can detect by classical statistical hypothesis tests.

The two most popular statistical tests used to judge if two samples of quality indicator values $I_{i, \text { and }} I_{j,}$, from two different algorithms $a_{i}$ and $a_{j}$ differ in their location (e.g. expected value) are the $t$-test and the Wilcoxon rank sum test [9]. If we were to use statistical hypothesis tests in a rigorous way to decide which algorithm is best, we would need to adjust the significance level $\alpha$ for the number of tests being performed. Since we are using the test result only as a substitute for the relevance of a result, we will not worry about controlling the global significance level. This leads us to the following new comparison operator for the $t$-test in the average quality scenario:

$$
a_{i} \succ a_{j} \Leftrightarrow p_{i j}^{t} \leq \alpha \wedge \bar{I}_{i}>\bar{I}_{j} \quad a_{i} \sim a_{j} \Leftrightarrow p_{i j}^{t}>\alpha,
$$

where $p_{i j}^{t}$ is the $p$-value of the $t$-test. 
The Wilcoxon rank sum test is based on the operator

$$
a_{i} \succ a_{j} \Leftrightarrow p_{i j}^{U} \leq \alpha \wedge \tilde{U}>0.5 \quad a_{i} \sim a_{j} \Leftrightarrow p_{i j}^{U}>\alpha,
$$

where $\tilde{U}$ is the test statistic and $p_{i j}^{U}$ the corresponding $p$-value.

Both rules are reflexive, complete but not antisymmetric. In the $t$-test case, we would need to assume equal variances. This seems highly unrealistic and might suggest that the procedure is not useful for any real world problems, but in practice the procedure is usually transitive which justifies its application.

For the Wilcoxon rank sum test there are several conditions under which it is transitive. For continuous distributions they usually reduce to the assumption that the density function is symmetric. Again, this is not necessarily realistic since we may assume that the distribution of our quality indicator has an upper bound.

There exists a wealth of literature on deriving transitive relations from intransitive relations in the context of social choice theory $([10])$. The resulting transitive relation would then be a partial ordering of the algorithms.

\section{Consensus ranking}

If we have a single function $f$ and a single quality indicator $I$ we can use one of the comparison operators from the previous section, depending on our scenario, to derive a (partial) order of the algorithms. Sadly, most real world situations are not that easy. Often we will have numerous, if not an infinite number of functions, representing our problem domain. At the same time, we may be interested in more than one quality indicator. If we want to find the best algorithm in such a situation, we need to first reduce it into a set of benchmark scenarios for which we already know how to infer a ranking.

First, we need to choose a fixed set of test problems $\mathcal{F}=\left\{f_{1}, \ldots, f_{b}\right\}$ which represents our problem domain. In choosing the $f_{i}$, it is crucial that we uniformly sample from the set of all possible functions. Then we need to define one or more suitable quality indicators and corresponding comparison operators for each test problem. Denote these by $\mathcal{V}=\left\{V_{1}, \ldots, V_{q}\right\}$. Combining these will give us a set of benchmark scenarios we are interested in. We will denote the $b q$ rankings resulting from the analysis of these benchmark scenarios by $r_{i, j}$ for the ranking induced by the $i$-th function and the $j$-th comparison operator. Let us call the resulting set of rankings $\mathcal{R}$. 
What we now have are $b q$ different opinions on which algorithm is best. What we need is a consensus among these opinions. We have seen in the previous section that depending on our benchmarking scenario we need different comparison operators which may lead to different rankings of the algorithms. This is to be expected since we are ranking them based on different definitions on what we consider to be a better algorithm. In this section, we will see that there are different methods for deriving a consensus from our set of rankings $\mathcal{R}$. These methods do not coincide with a benchmarking scenario. Instead, they offer different tradeoffs between properties that the consensus fulfills. So, before we have seen the first consensus method we need to accept the fact that from this point forward, we cannot objectively define the best algorithm. Instead, our statement of which algorithm is best depends on our subjective choice of a consensus method.

\subsection{Consensus Criteria}

In order to ease the choice of a consensus method, let us begin by defining some criteria that a consensus method should meet [5]:

Criterion 1 A consensus method $\mathrm{cm}$ that takes into account all rankings instead of mimicking one predetermined ranking is said to be non-dictatorial.

Criterion 2 A $\mathrm{cm}$ that, given a fixed set of rankings, deterministically returns a complete ranking is called a universal consensus method or is said to have a universal domain.

Criterion 3 A cm fulfills the independence of irrelevant alternatives criterion, short IIA criterion, if given two sets of rankings $\mathcal{R}=\left\{r_{1}, \ldots, r_{n}\right\}$ and $\mathcal{S}=\left\{s_{1}, \ldots, s_{n}\right\}$ in which for every $i \in\{1, \ldots, n\}$ the order of two algorithms $a_{1}$ and $a_{2}$ in $r_{i}$ and $s_{i}$ is the same, the resulting consensus rankings rank $a_{1}$ and $a_{2}$ in the same order.

IIA means that introducing a further algorithm does not lead to a rank reversal between any of the already ranked algorithms which is a very strict requirement. In fact the next criterion is incompatible with IIA

Criterion $4 \mathrm{~A} \mathrm{~cm}$ which ranks an algorithm higher than another algorithm if it is ranked higher in a majority of the individual rankings, fulfills the majority criterion.

Criterion $5 \mathrm{~A} \mathrm{~cm}$ is called Pareto efficient if given a set of rankings in which for every ranking an algorithm $a_{i}$ is ranked higher than an algorithm $a_{j}$, the consensus also ranks $a_{i}$ higher than $a_{j}$ 
No consensus method can meet all of these criteria because the IIA criterion and the majority criterion are incompatible. But even if we ignore the majority criterion, there is no consensus method which fulfills the remaining criteria [5]. So if we choose different criteria that our consensus method should meet, we may get very different consensus rankings.

\subsection{Positional methods}

In a positional consensus method we need a function $s$ which, given a ranking $r$ and an algorithm $a_{i}$, returns the score of $a_{i}$ under ranking $r$. We can then calculate the sum of the scores for each algorithm as follows

$$
s_{i}=\sum_{k=1}^{b} \sum_{l=1}^{p} s\left(a_{i}, r_{k, l}\right)
$$

We then derive the consensus ranking by ordering the algorithms according to their scores

$$
a_{i} \succ a_{j} \Longleftrightarrow s_{i}>s_{j} \quad a_{i} \sim a_{j} \Longleftrightarrow s_{i}=s_{j}
$$

This leaves us with the choice of a suitable score function. The simplest possible choice would be

$$
s\left(a_{i}, r\right)=\left\{\begin{array}{ll}
1 & \text { if in } r a_{i} \succ a_{j} \text { for all } j \\
0 & \text { otherwise }
\end{array} .\right.
$$

With this score function the best algorithm in each scenario gets one vote and all other algorithms do not. However, this introduces bias into the consensus.

Instead we will consider the following function

$$
s^{B C}\left(a_{i}, r\right)=\sum_{i \neq j} \mathbf{I}\left(a_{i} \succ a_{j}\right)
$$

It counts the number of algorithms that are not better than the algorithm whos score we are calculating. So an algorithm gets one point for each algorithm that it weakly dominates.

The function $s^{B C}$ leads to one one of the oldest consensus methods [11] namely the Borda count method. This is the optimal consensus method under all positional consensus methods [12]. While optimal in some sense, the Borda method lacks mathematical rigor. It seems like an ad hoc definition without any theoretical justification. The next approach to consensus ranking is exactly the opposite. 


\subsection{Optimization based methods}

Assume we have a set of candidate consensus rankings $\mathcal{C}$. This could be the set of all linear, partial or weak orders of our $k$ algorithms or some reasonable subset of these. Further assume we have a function

$$
d: \mathcal{T} \times \mathcal{T} \rightarrow \mathbb{R}^{+},
$$

where $\mathcal{T}:=\mathcal{R} \cup \mathcal{C}$, with the following properties

- Non-negativity: For any $t_{1}, t_{2} \in \mathcal{T}, d\left(t_{1}, t_{2}\right) \geq 0$ and $d\left(t_{1}, t_{2}\right)=0$ iff $t_{1}=t_{2}$.

- Symmetry: For any $t_{1}, t_{2} \in \mathcal{T}, d\left(t_{1}, t_{2}\right)=d\left(t_{2}, t_{1}\right)$.

- Triangle inequality: For any $t_{1}, t_{2}, t_{3} \in \mathcal{T}, d\left(t_{1}, t_{2}\right)+d\left(t_{2}, t_{3}\right) \geq d\left(t_{1}, t_{3}\right)$ and equality only holds iff $t_{2}$ lies between $t_{1}$ and $t_{3}$.

Then $d$ is a measure for how far apart two rankings are. What is missing is a notion of betweenness. We will define this in terms of pairwise comparisons. We will say $t_{2}$ lies between $t_{1}$ and $t_{3}$ if for all pairs of algorithms either $t_{2}$ agrees with $t_{1}$ or $t_{3}$ on the relative order of the pair, or $t_{1}$ and $t_{3}$ have conflicting orderings for the pair and $t_{2}$ declares the pair to be tied.

Using this distance measure, we could transform our consensus ranking problem into an optimization problem

$$
\arg \min _{c \in \mathcal{C}} \sum_{i=1}^{b} \sum_{j=1}^{q} d\left(r_{i, j}, c\right)^{l} \quad l \geq 1 .
$$

The consensus ranking is then given by the ranking whose median (for $l=1$ ) or mean (for $l=2$ ) distance to all benchmark scenario rankings is minimal.

We will only review one distance function (see [10] for an overview) and motivate it by postulating a set of axioms which it should satisfy [13]. The first axiom simply states that a permutation of the labels should not change the distance between two rankings. The second axiom states that if the two rankings differ only on a subset of all algorithms that form a segment of the rankings, then the distance between the two rankings should be equal to the distance between the two differing segments. And finally we need one axiom to fix the scale. It simply states that the minimum distance between two different rankings is 1 .

Kemeny and Snell [13] showed that these three axioms and the metric properties uniquely describe a single distance function, the symmetric difference or short SD. It is defined as

$$
d^{S D}\left(t_{1}, t_{2}\right):=\overrightarrow{1}^{\prime}\left|\vec{I}^{t_{1}}-\vec{I}^{t_{2}}\right| \overrightarrow{1}
$$


where $\overrightarrow{1}$ is the $k$ dimensional one vector, $\vec{I}^{t_{i}}$ the incidence matrix belonging to the relation that corresponds to the ranking $t_{i}$ and $|\cdot|$ denotes the element wise absolute value. It counts how many cases there are where $a_{i} \succ a_{j}$ is contained in one of the relations but not the other. Thus, the optimization problem from above tries to minimize the average number of rank reversals in the consenus ranking.

When we talk about a symmetric difference consensus we will denote it by $\mathrm{SD} / \mathcal{C}$ where we replace $\mathcal{C}$ with $\mathrm{L}$ for the set of all linear orders and $\mathrm{O}$ for the set of all partial orders.

\subsection{Comparison}

So far we have introduced two consensus methods. The SD/L and SD/O methods meet the majority criterion, the Border count (BC) method does not.

$\mathrm{SD} / \mathrm{L}$ and SD/O fulfill the majority criterion, therefore they cannot fulfill the IIA criterion. However, on real data they seldom show rank reversals if algorithms are added or dropped from the rankings [14]. Sadly, the Borda count method also fails the IIA. A thorough investigation and comparison of the Borda method and the SD method is given in [14]. Two remarkable results proven there are that the SD method always ranks the Borda winner above the Borda loser and that the Borda method always ranks the SD winner above the SD loser.

To summarize, if we want to find a best algorithm for a problem domain, we need to uniformally pick a set of test functions from the problem domain. These combined with a quality indicator and corresponding comparisons operator define our benchmark scenarios and induce a set of partial or linear orders of our algorithms. Using consensus methods, we can combine these rankings into a final order. The choice of consensus method is crucial and at the same time highly subjective. There is no right way to choose and therefore no one best algorithm. Our choice of algorithm will always depend on our choice of consensus method.

\section{Experimental Results}

This section will illustrate two possible benchmarking scenarios using a publicly available dataset from the CEC 2007 competition [15]. We will see that there is no such thing as the 'best' algorithm. Instead, we can only gain insight into the relative strengths and weaknesses of the different procedures.

The stated goal of the competition was to evaluate how well the entries meet the following three objectives 


\begin{tabular}{lcccc}
\hline Function & Objectives & Separable & Unimodal & Geometry \\
\hline oka2 & 2 & partial & partial & concave \\
sympart & 2 & no & no & concave \\
s_zdt 1 & 2 & yes & yes & convex \\
s_zdt2 & 2 & yes & yes & concave \\
s_zdt 4 & 2 & yes & partial & convex \\
r_zdt 4 & 2 & no & no & convex \\
s_zdt6 & 2 & yes & no & concave \\
s_dtlz2 & $3 ; 5$ & yes & yes & concave \\
r_dt lz2 & $3 ; 5$ & no & no & concave \\
s_dt lz3 & $3 ; 5$ & yes & no & concave \\
wfg1 & $3 ; 5$ & yes & yes & mixed \\
wfg8 & $3 ; 5$ & no & yes & concave \\
wfg9 & $3 ; 5$ & no & no & concave \\
\hline
\end{tabular}

Table 2: Properties and number of objectives used of the 13 test functions chosen for the CEC2007 EMOA competition.

- maximize well defined performance measures,

- scale with the number of objectives,

- scale with the number of parameters.

In order to assess the quality of each algorithm, a set of test functions over which to formulate the optimization problems was chosen (Table 2).

The domain of problems that these test functions try to cover is not stated. Judging by their diversity, an attempt was made to cover all possible aspects of multiobjective optimization. While the choices are fairly balanced when it comes to being separable (yes: 7 , no: 5 , mixed: 1) and unimodal (yes: 5, no: 6, partial: 2) some combinations are nevertheless missing, e.g. a nonseperable multimodal function with a convex Pareto front. If there are interactions between the different characterizations, then it would be desirable to extend the set of test functions to include examples from all possible characterizations. This would in fact be equivalent to a full factorial experimental design. Using these 13 test functions, given objective dimensions and budgets of 5000,50000 , and 500000 function evaluations, 57 unique multiobjective optimization problems were constructed. 


\begin{tabular}{ll}
\hline Algorithm & Description \\
\hline demowsa & Differential Evolution with Self Adaption \\
gde3 & Generalized Differential Evolution 3 \\
mo_de & MO Differential Evolution \\
mo_pso & MO Particle Swarm Optimization \\
mosade & Self adapting Differential Evolution \\
nsga2_pcx & NSGA-II variant using PCX variation \\
nsga2_sbx & NSGA-II variant using SBX variation \\
mts & Multiple Trajectory Search \\
\hline
\end{tabular}

Table 3: Contestants in the CEC2007 EMOA competition.

The last ingredient for the competition are criteria by which to measure how well each algorithm approximated the Pareto front. Here, the dominated hypervolume difference indicator $\left(I_{H V}\right)$ and the $R 2$ indicator $\left(I_{R 2}\right)$ were used. For the hypervolume indicator a reference point was provided for each problem definition. A reference set was also provided for each test problem. In the results section we will see that some hypervolume indicator values are negative. This leads us to believe that a better reference set could have been provided.

Eight contestants took part in the competition (see http://www3.ntu.edu.sg/home/ EPNSugan/ for details), which are given in Table 3.

\subsection{Results}

Each contestant was asked to perform 25 independent runs on each problem and report minimum, median, maximum, mean and variance of each performance measure over the 25 runs. They where also asked to report how they tuned the algorithms and how many function evaluations were necessary for this step. We will only consider the raw data and will not take into account how much effort each team spent tuning the hyperparameters of their algorithm.

Many algorithms nearly have no variation in quality for some test problems while others have consistently high variation. For some test problems and quality indicator combinations, it is hard to see if there truly is a best algorithm. An example is $s_{-} d t l z 2$ with a 3 dimensional objective space, 500000 function evaluations and the R2 quality indicator, which is visualized in Figure 1. The dot represents the mean performance of the algorithm on the optimization problem and the line marks the range of the quality indicator values. 


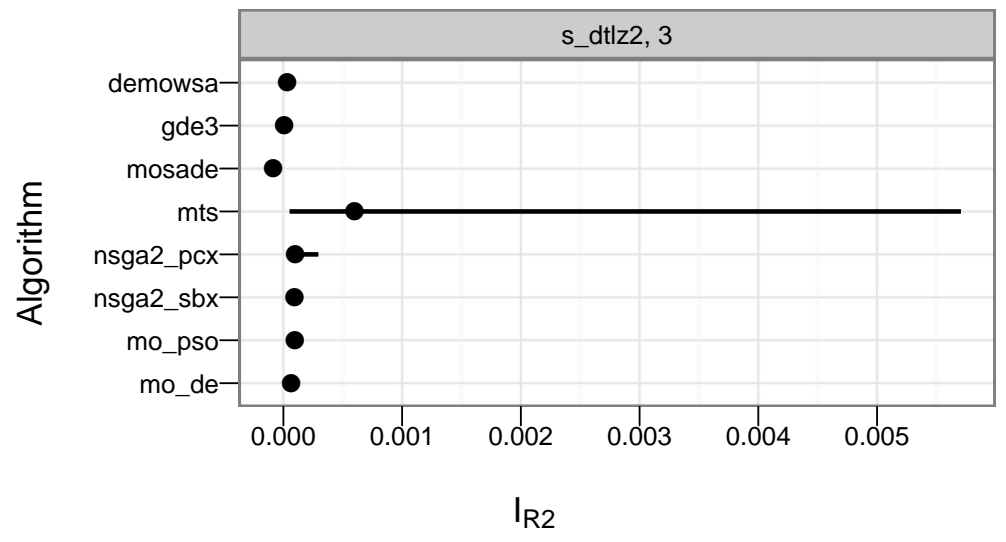

Figure 1: CEC2007 EMOA competition results for 500000 function evaluations and the R2 quality indicator.

Here, all algorithms except mts seem to be tied for first place. If we look at the mean ranking of this benchmark scenario, we get

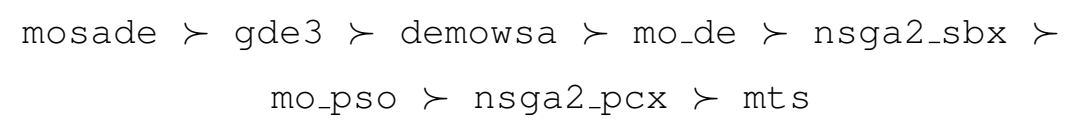

If we employ a $t$-test based relevance ranking with $\alpha=0.05$, we get the following result

mosade $\succ$ gde $3 \succ$ demowsa $\succ$ mo_de $\succ$ [mo_pso $\sim$ mts $\sim$ nsga2_pcx $\sim$ nsga2_sbx]

Here, we see that the last four algorithms are tied and a fallacy of the $t$-test approach. If the variance of the data is low enough it will still declare one algorithm better than another even though there is only a statistically significant, but not scientifically relevant difference.

We will be using two different ranking operators, one is the mean ranking $r^{m}$ and the other a $t$-test based relevancy ranking $r^{r}$. A Wilcoxon rank sum based relevancy ranking operator is not possible as we do not have the original data. Thus, we are looking for a good average performer. Because of the way $r^{r}$ is defined, it does not change the order of the algorithms compared to $r^{m}$, it only introduces ties to account for the uncertainty.

Why do we use a $t$-test without knowing that the data are really normal? Figure 2 shows a density estimate of the distribution of the difference between the mean and median quality indicator values by quality indicator and number of function evaluations. All of these distributions clearly peak around zero and have very few extreme values, so we have no reason to believe that 

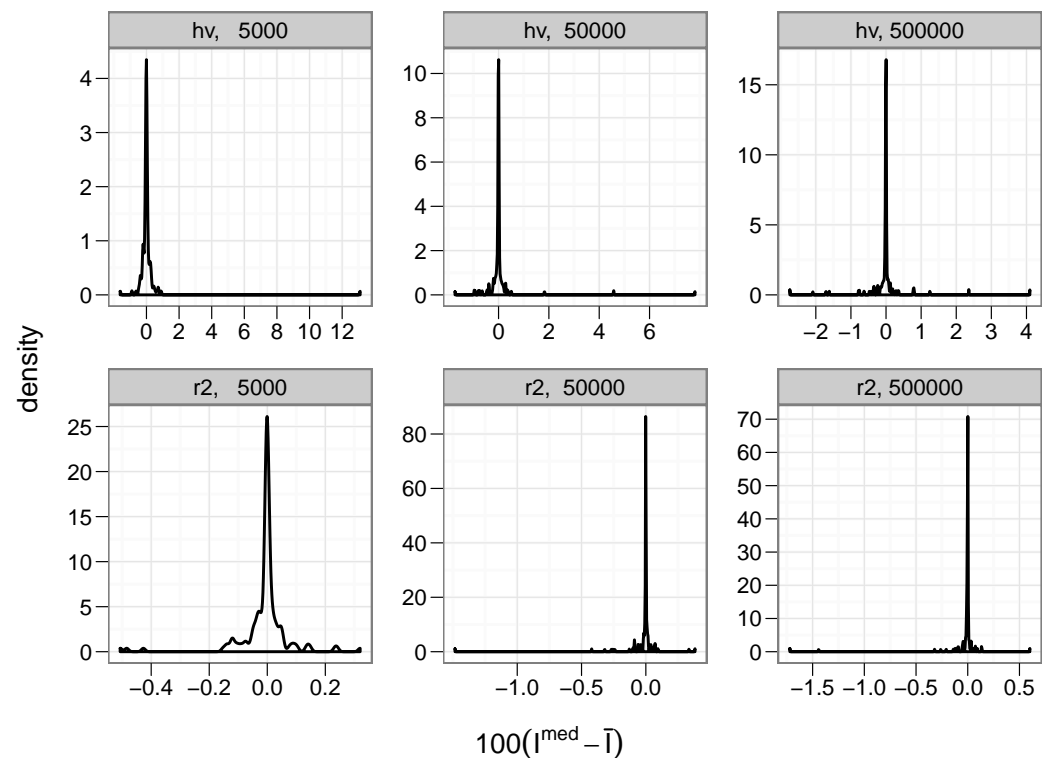

Figure 2: Density estimates of the difference between the median and mean quality indicator by metric and number of function evaluations. Note the change in scale to increase readability.

the distributions of the quality indicators' means are not symmetric. Furthermore, we have 25 observations, so that the central limit theorem tells us that we are well on our way to normality. One thing we need to check before we can use the rankings produced by $r^{r}$ is if they are all transitive. This is the case, so all of the rankings are weak orderings of the algorithms. Of the 114 rankings 32 are strict rankings, meaning they are a linear order of the algorithms, which means that $r^{r}$ introduces ties into 82 of the rankings. This may lead us to believe that the mean rankings are not very accurate. As we will later see, this does not seem to be the case because most of the consensus rankings over the two sets of rankings are in agreement.

We will use both consensus methods introduced to compare and contrast some of the results. They will be indicated by $\mathrm{cm}_{B C}$ for the Borda count consensus and $\mathrm{cm}_{S D / L}$ for the SD/L consensus. The original analysis of the competition used a mean ranking operator and a consensus method that is equivalent to the Borda count method. The overall ranking that results under this analysis regime is

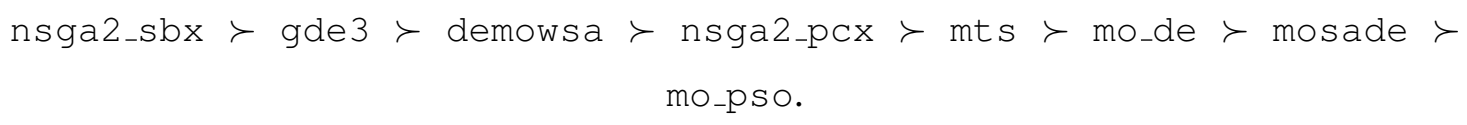


If we use the relevance rankings $r^{r}$ instead, we get

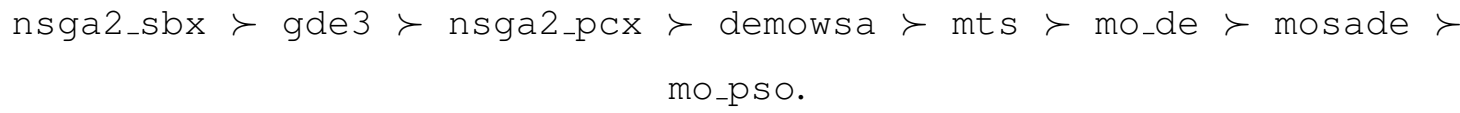

Here nsga2_pcx and demowsa reverse their relative positions but the overall picture looks quite similar.

If we look at the $\mathrm{SD} / \mathrm{L}$ consensus rankings we get

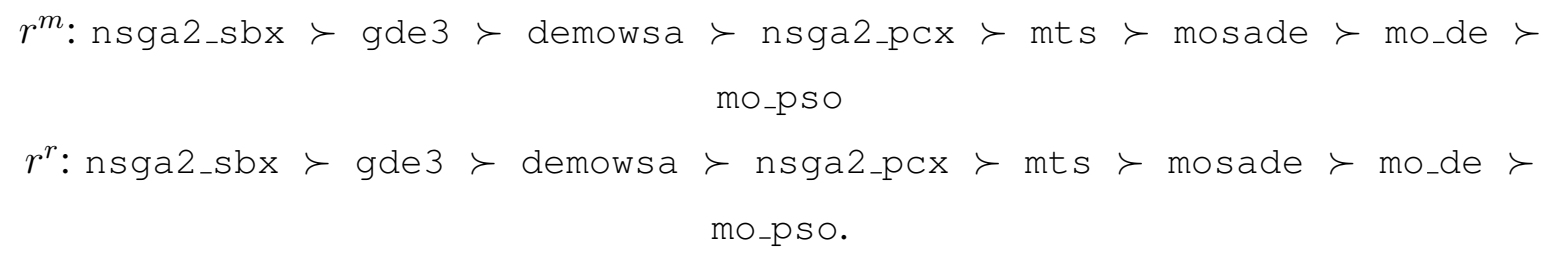

Here the positions of mo_de and mosade are reversed when compared to the Borda consensus. This is interesting, since we know that the Borda method does not adhere to the IIA criterion and mo_pso is an irrelevant alternative to the two, but was submitted by the same authors who submitted mo_de.

By eliminating the irrelevant alternative mo_pso the Borda method now ranks mo_de as last instead of as second to last, as we might have suspected. SD/L does not change its ranking. This is an example of how susceptible the Borda method really is to irrelevant alternatives while $\mathrm{SD} / \mathrm{L}$ is relatively robust against such phenomenon even though it also does not fulfill the IIA criterion.

Instead of continually listing all four rankings in every following situation we will only look at one ranking if they do not differ significantly.

One of the first things we might be interested in is what the consensus rankings look like if we calculate them separately for each quality indicator. And in fact they do differ as can be seen in Table 4. This is also the first time that we do not have a unique consensus. The SD/L method gives us two possible consensus rankings when looking at the dominated hypervolume consensus ranking of the relevance rankings $r^{r}$.

We will continue by looking at the overall distribution of the ranks for each algorithm. These are depicted in Figure 3. We can clearly see why nsga2_sbx is ranked first in almost all consensus rankings we have looked at. In both cases almost 50 of the 114 rankings show it to be the best algorithm of all. The only other algorithm that is consistently ranked above average is gde 3. All others are either ranked below average in the majority of the benchmark scenarios (mo_pso, mo_de) or have an almost uniform distribution over all ranks. This suggests that these 


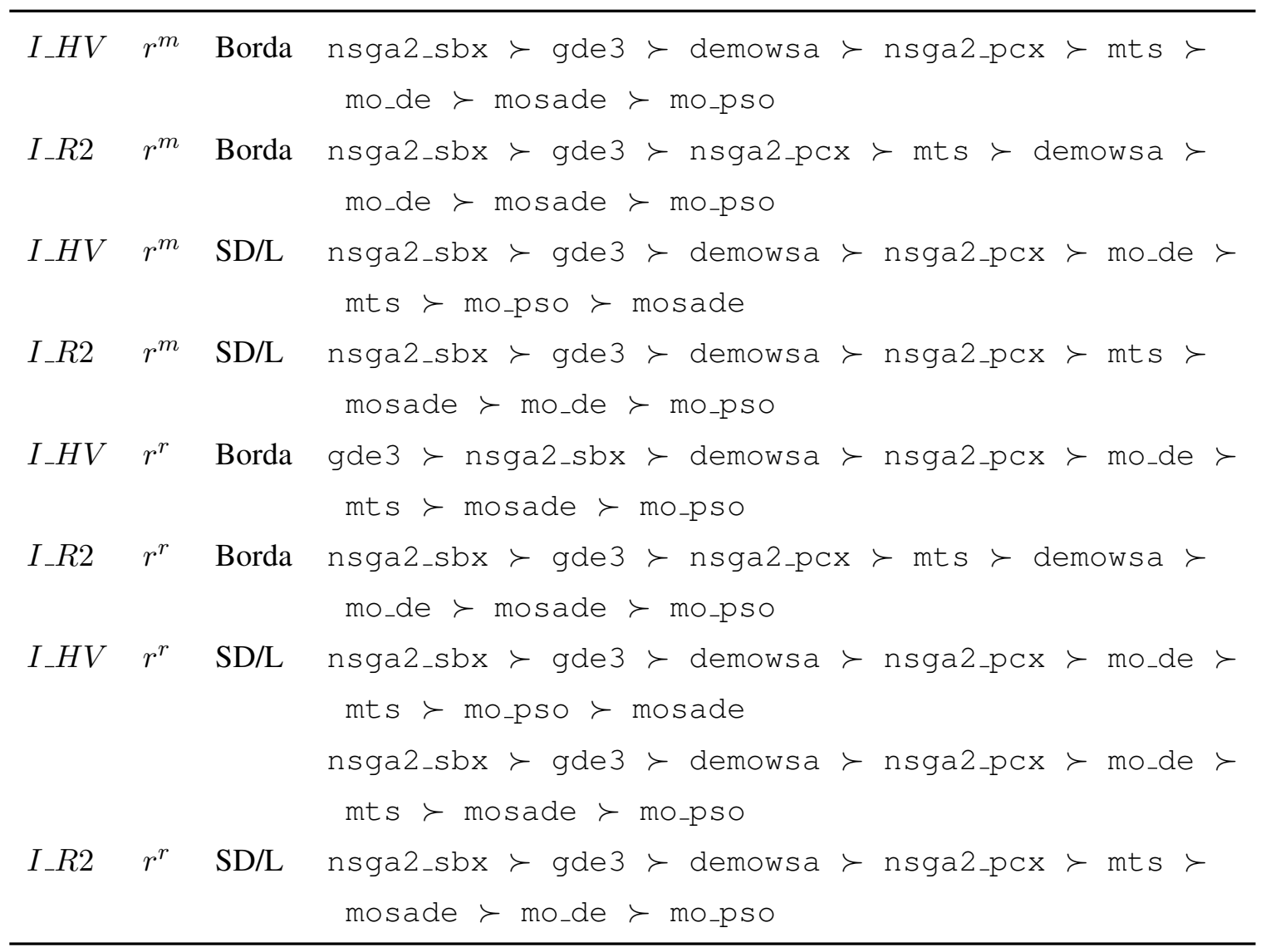

Table 4: Consensus rankings by quality indicator for each combination of ranking method and consensus method.

algorithms perform well on a subset of all test functions from our domain. Further drill down in the data does not reveal any evidence that the last statement is true. This could have different reasons. For example, we might be missing some variable that divides the test problems into 'good' or 'bad' groups.

We defined a distance measure on rankings for the SD/L consensus method. Using this distance measure we can calculate the pairwise distance between all rankings and apply a Multidimensional scaling (MDS) to $r^{m}$. In general, the distance between the ranking using the HV indicator and the ranking using the $R 2$ indicator is small. We might question why we should use both indicators simultaneously. Furthermore the Walking Fish Group test problems as well as the $s_{-} z d t$ class of test problems do not induce much difference regarding the consensus rankings. Thus one representative from both classes would have been sufficient. 


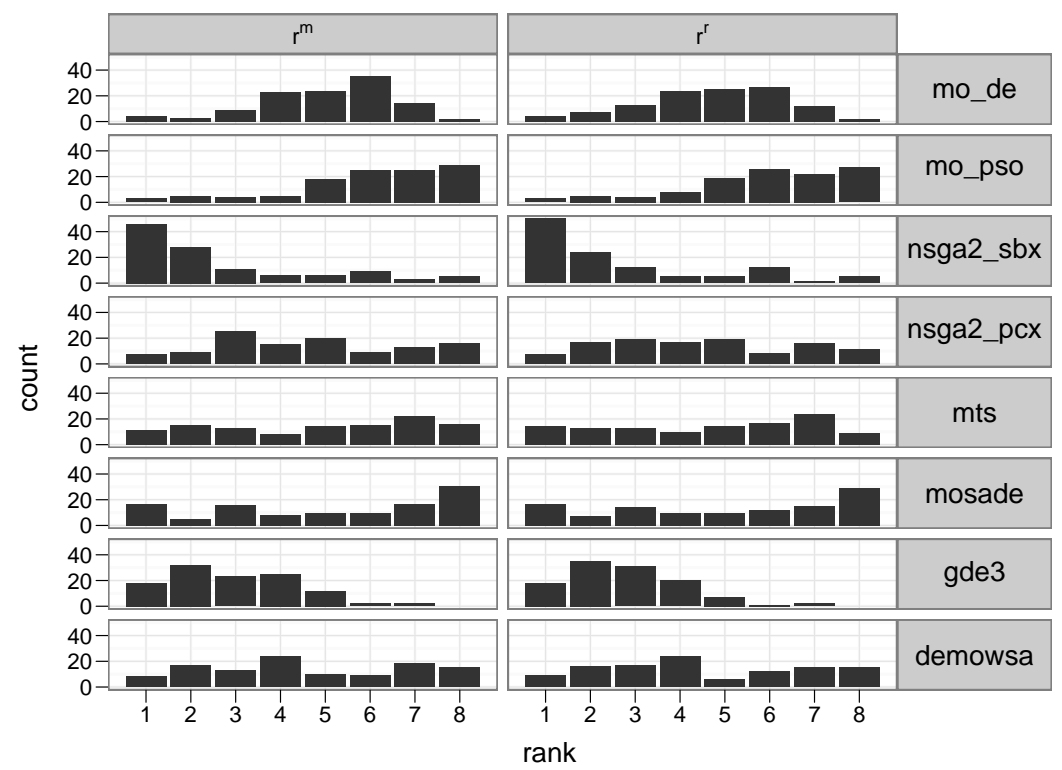

Figure 3: Distribution of the ranks for all rankings using the mean ranking operator and the relevance ranking operator.

\section{Conclusion and Outlook}

We have developed a framework to systematically compare evolutionary multiobjective optimization algorithms under different settings. We then introduced methods to combine these rankings from different benchmark scenarios into a consensus ranking to choose the 'best' algorithm. Here, we saw that there is no such thing as a universally best consensus and therefore there can be no best algorithm for everyone. To illustrate this we analyzed a competition dataset from the CEC 2007 EMOA competition.

All of the methods shown are either implemented in the R-package emoa or can easily be constructed from the functions contained in the emoa and relations packages. In the future, we would like to study other ranking operators as well based on parametric models of the data. Examples would include the Bradley-Terry model also used by [2] to estimate the abilities of machine learning algorithms. Furthermore, a comprehensive survey of voting literature could reveal more criteria which might ease the choice of a consensus method.

In addition, benchmarking is a very important issue for single-objective evolutionary optimization as well. Modifications of the proposed methodology itself are not required. It will be investigated which performance indicators are suited best for deriving consensus rankings for single-objective evolutionary algorithms and which kind of test functions form a representative 
testset in order to perform benchmarking studies in a systematic and statistically sound way. Lastly a research group in Munich has started working on new graphical representations of rankings [16].

\section{Acknowledgment}

This work was supported by the Collaborative Research Center SFB 823 of the German Research Foundation. Special thanks to Prof. Dr. Günter Rudolph for his constructive comments.

\section{References}

[1] J. Knowles, L. Thiele, and E. Zitzler, "A Tutorial on the Performance Assessment of Stochastic Multiobjective Optimizers,' Computer Engineering and Networks Laboratory, ETH Zurich, TIK Report 214, 2006.

[2] K. Hornik and D. Meyer, "Deriving consensus rankings from benchmarking experiments," in Advances in Data Analysis (Proc. of the 30th Ann. Conf. of the Gesellschaft für Klassifikation, R. Decker and H.-J. Lenz, Eds. S Springer, Berlin, 2007, pp. 163-170.

[3] O. Mersmann, emoa: Evolutionary Multiobjective Optimization Algorithms, 2009, R package version 0.1-0. [Online]. Available: http://www.statistik.tu-dortmund.de/ olafm/ emoa/

[4] K. Hornik and D. Meyer, "Relations: Data structures and algorithms for relations," 2009, $\mathrm{R}$ package version $0.5-4+$ modifications.

[5] K. J. Arrow, "A difficulty in the concept of social welfare," Journal of Political Economy, vol. 58, p. $328,1950$.

[6] D. J. Hunter, Essentials of Discrete Mathematics. Jones and Bartlett Publishers, Boston, MA, 2008.

[7] C. A. Coello Coello, D. A. V. Veldhuizen, and G. B. Lamont, Evolutionary Algorithms for Solving Multi-Objective Problems. Kluwer Academic Publishers, New York, 2002.

[8] E. Zitzler and L. Thiele, "Multiobjective optimization using evolutionary algorithms - A comparative case study," in Parallel Problem Solving from Nature V, E. A. Eiben et al., Eds., Springer, Berlin, 1998, pp. 292-301. 
[9] A. Mood, F. Graybill, and D. Boes, Introduction to the Theory of Statistics. McGraw-Hill, New York, 1974.

[10] W. D. Cook and M. Kress, Ordianl Information \& Preference Structures. Prentice Hall, Upper Saddle River, NJ, 1992.

[11] J. C. de Borda, "Mémoire sur les Élections au scrutin," Historie de l'Académie Royale des Sciences, 1781.

[12] D. G. Saari, "The optimal ranking method is the borda count," Northwestern University, Center for Mathematical Studies in Economics and Management Science, Discussion Papers 638, 1985. [Online]. Available: http://ideas.repec.org/p/nwu/cmsems/638.html

[13] J. G. Kemeny and J. L. Snell, Mathematical Models in the Social Siences. MIT Press, Cambridge, MA, 1972.

[14] D. G. Saari and V. R. Merlin, “A geometric examination of kemeny's rule," Social Choice and Welfare, vol. 17, p. 2000, 1997.

[15] V. L. Huang, A. K. Qin, K. Deb, E. Zitzler, P. N. Suganthan, J. J. Liang, M. Preuss, and S. Huband, "Problem definitions for performance assessment on multi-objective optimization algorithms,” Nanyang Technological University, Tech. Rep., January 2007.

[16] M. J. A. Eugster, T. Hothorn, and F. Leisch, "Exploratory and inferential analysis of benchmark experiments," Ludwigs-Maximilians-Universität München, Department of Statistics, Tech. Rep. 30, 2008. 


